\title{
Design and Development of Cost Effective Automatic Machine for Powder Packaging
}

\author{
M. R. Saraf ${ }^{*}$, V. V. Ruiwale ${ }^{\dagger}$, V. V. Kulkarni ${ }^{\dagger}$ and S. M. Kulkarni ${ }^{\dagger}$ \\ †Mechanical Engineering Department, MITCOE, Sri Savitribai Phule Pune University, Pune, Maharashtra, India \\ Accepted 02 March 2016, Available online 15 March 2016, Special Issue-4 (March 2016)
}

\begin{abstract}
The packaging industry has advanced leaps and bounds over the few years. The pouch packaging machines also known as Form Fill and Seal Machines (FFS Machines) are available over a wide capacity range. A low cost automated packing machine can be used by small enterprises which would help reduce their cost of plant. This low cost automated machine uses simple pneumatic, mechanical and electric systems. In this paper we have presented one such low cost pouch filling machine. An additional weighing and pouring mechanism has been added to increase the accuracy of the system. The process flow has been described in detail. Various processes involved in the pouch packaging are neatly aligned and properly timed to get optimum production rate. A mechatronics system, developed for this machine, which takes feedback from sensors and accordingly controls the manipulators has been introduced in this paper. A microcontroller system is used for this particular machine. A detailed cost comparison between a conventional machine and the one developed by us has been presented.
\end{abstract}

Keywords: Low Cost Automation, Microcontroller, PLC

\section{Introduction}

In the current global scenario better quality, low cost with higher productivity are major factors for any enterprise to flourish. To cope up with the ever increasing market competition, low cost automation is the safest strategy. Low Cost Automation comprises the use of standard components to mechanize or automate machines, processes and systems. These low cost automated machines can be operated by semi-skilled or unskilled labors as it requires limited human intervention. In this paper need of automation, types of automation, design, process flow and cost comparison have been described.

\section{Literature Review}

Pouch packing machines are commonly known as Form Fill and Seal Machines. There are classified as two main types- Vertical Form Fill and Seal Machines and Horizontal Form Fill and Seal Machines. Vertical Form Fill and Seal Machines (VFFS Machine) are used to pack regular shaped solid products or liquids while, Horizontal Form Fill and Seal Machines are used to pack irregular shaped products. In this paper we focus on the Vertical Form Fill and Seal Machines. VFFS machines are widely used in most of the industries to pack products of various sizes (depending on their weights).

*Corresponding author: M. R. Saraf

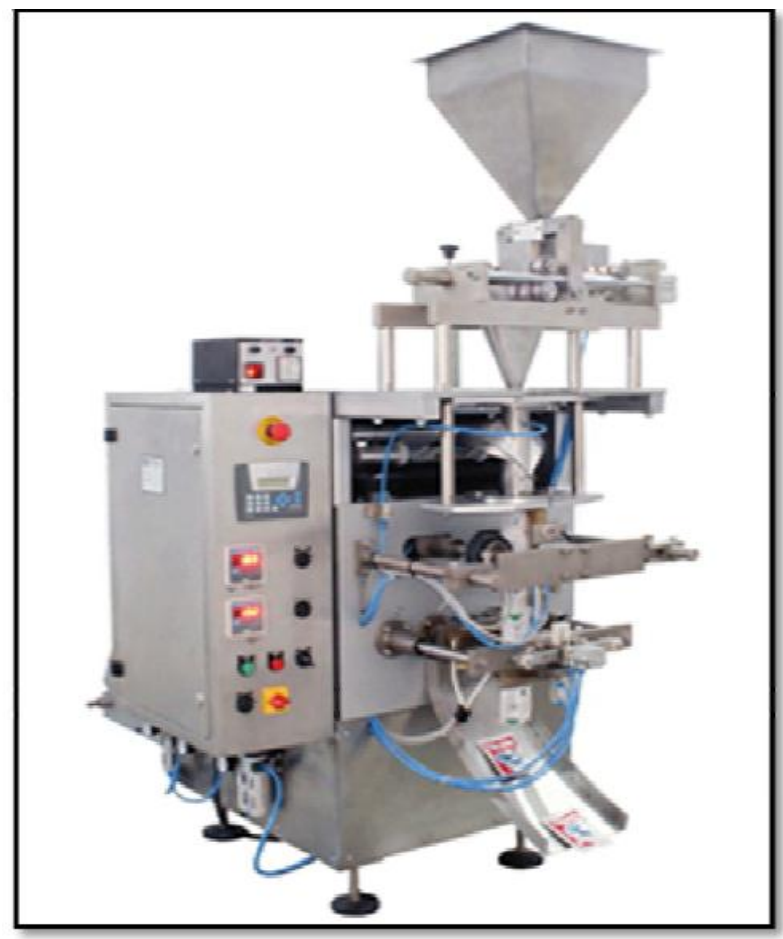

Figure 1. Conventional System

The VFFS Machines are again classified in two waysAccording to their type of motion (Continuous motion and Intermittent motion) and According to their type of automation (Fully automated and Semi automated). In making of the pouch two seals have to be made 
horizontal and vertical as it will be further explained in detail. Intermittent motion machine makes the vertical seal while the film is in motion but for the horizontal seal the film stops whereas, the continuous motion machine makes the vertical and the horizontal seal without stopping the film. Hence, higher production rates can be achieved by the continuous motion machine. On the other hand, Fully automated machine requires no human intervention while Semi automated machines require humans to perform some part of packing operation. The machine discussed in the paper can be classified as an intermittent motion machine or fully Automatic machine.

\section{Need of Automation}

Pouch packing involves a number of steps like bag forming, weighing, and sealing the bag. If all these processes are to be done manually, the time required is more. The production rates will be slow. In order to achieve higher production rates use of automated machines is necessary. Automated machines are time savvy which gives the entrepreneurs to gain a competitive edge. The quality of the work is high and is repetitive. Automated machines can help reduce the waste generated hence, leading to proper utilization of the resources. Workers may suffer from fatigue which might lead to improper job quality. Use of an automated machine lessens the labour dependency of the enterprise. The key to achieve all of the above stated advantages while keeping the cost to be minimum is Low Cost Automation.

\subsection{Types of Automation Techniques}

A pouch packing machine can $^{[2]}$ be automated in different ways. The three main types of automation techniques are Relay Logic control, Programmable Logic Control (PLC) and Microcontroller technique. Relay Logic Control uses relays interconnected to each other by wires to operate. A relay logic control panels are very large, sometimes large enough to occupy a wall. The life of a relay is limited so some of them need to be replaced, in order to replace a relay the machine needs to be stopped and eventually the production. To discover an error is very tedious as it is a very complex system. Electricians need to be very skillful in finding errors. The control system is very inflexible so changes cannot be done easily. Due to all such reasons, relay logic control is obsolete.

A Programmable Logic Controller, PLC or Programmable Controller is a digital computer used for automation of electromechanical processes, such a control of machinery on factory assembly lines, amusement rides, or light fixtures. PLCs are used in many industries and machines. Unlike general-purpose computers, the PLC is designed for multiple inputs and output arrangements, extended temperature ranges, immunity to electrical noise, and resistance to vibration and impact. Programs to control machine operation are typically stored in battery-backed-up or non-volatile memory. Microcontroller is commonly used term for a small computer on a single chip, which contains the microprocessor, memory and other accessories. Microcontroller has reduced size and price in comparing with other devices. Microcontrollers very frequently have amount of accessories like analog-todigital converter, digital-to-analog converter, programmable timer and counter etc. Powerful and carefully chosen electronics embedded in the microcontrollers can

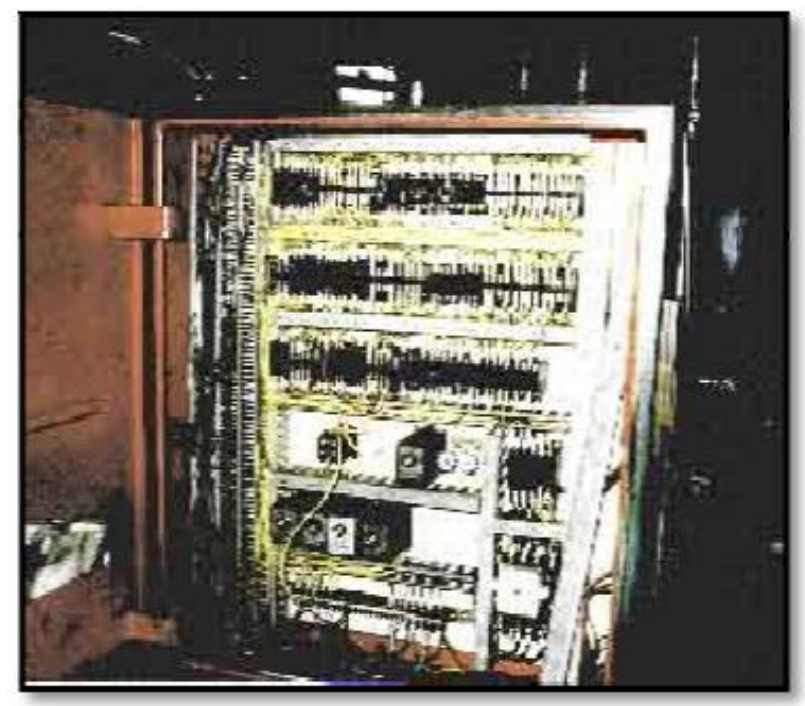

Figure 2. Relay Logic Control

PLC requires programming software, PLC control console and a computer to operate, whereas a microcontroller combined with some accessories can complete the job of automating a machine.

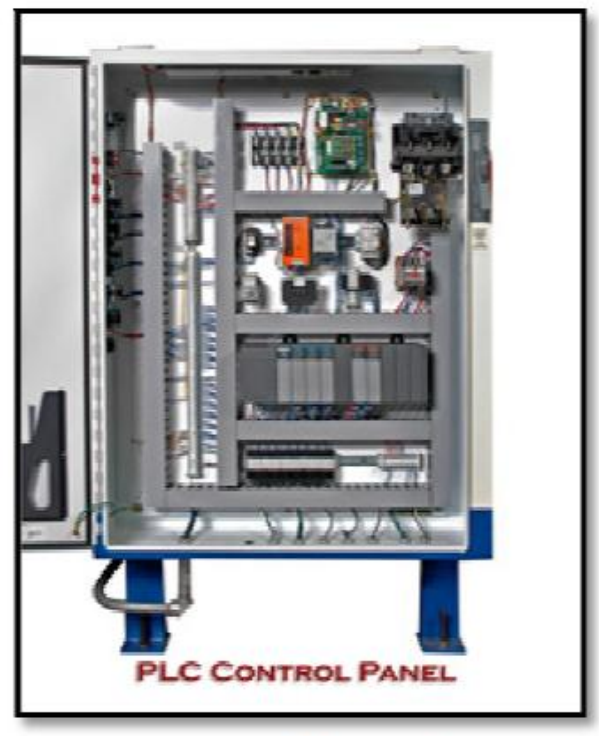

Figure 3. PLC control

The cost of PLC control is more than that of a microcontroller unit. PLCs are more flexible than microcontroller units but in this case, flexibility required is very less. Due to all such reasons, the microcontroller technique is much more suitable to 
achieve Low cost automated packing machine. Hence, in this case microcontroller technique is used.

\section{Principle of the System}

The material is fed into the hopper whichis situated on top of the machine. The hopper has a pipe attached to it at the bottom through which the material passes. The pipe has a flap arrangement to allow or prohibit the further flow of the material. The flap is actuated by a pneumatic cylinder, when the flap is open the material falls into a bowl. A load cell is placed beneath the bowl to measure the weight of the material falling in it. ${ }^{[4]}$

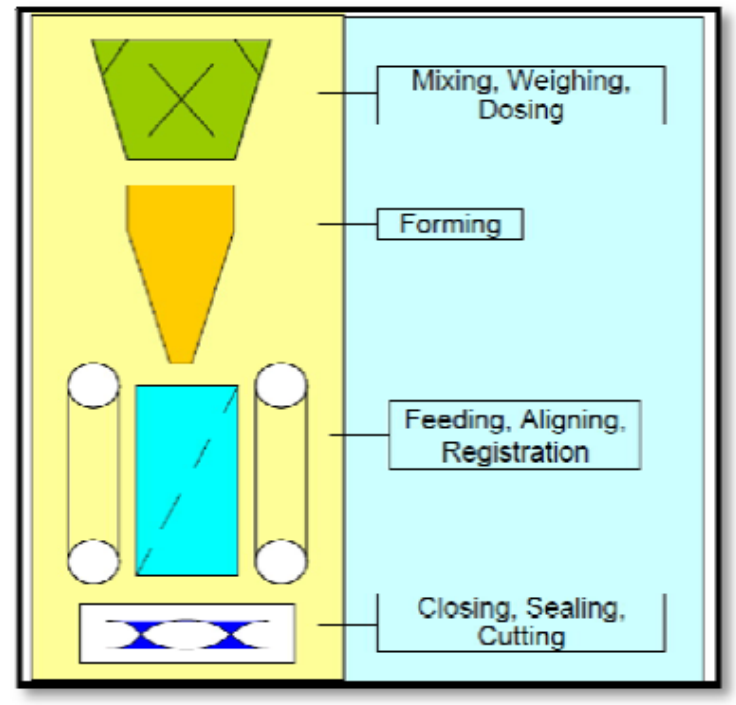

Figure 6. Block Diagram

When a specific amount of material falls into the bowl, flap is closed and the bowl rotates thus emptying it into the forming tube. The forming collar shapes the flat film into a round film tube. Low friction rollers guide the film to the forming area and maintain tension in the film thus preventing it from slagging. Vertical heat sealers seal the edges of the round film tube. Draw rollers pull the bag downwards after the vertical seal has been done which also unwinds the plastic film from the roll. Cross sealing jaws create top and bottom seals in the pouches. A cutter separates the pouches which is placed between the cross sealing jaws. Finally, an accurately weighed pouch is made.

\subsection{Machine Layout}

In the design shown above, a separate weighing mechanism is added. Load cell arrangement allows for accurate measurement of the material to be filled in the pouch. Load cell arrangement allows for accurate measurement of the material to be filled in the pouch. When the bowl is filled with the specific amount of material, it rotates and the material falls in the forming tube. A pneumatic cylinder and disc arrangement rotates the bowl. The forming collar is a uniquely designed structure by which the flat plastic film is turned into a vertical round film.

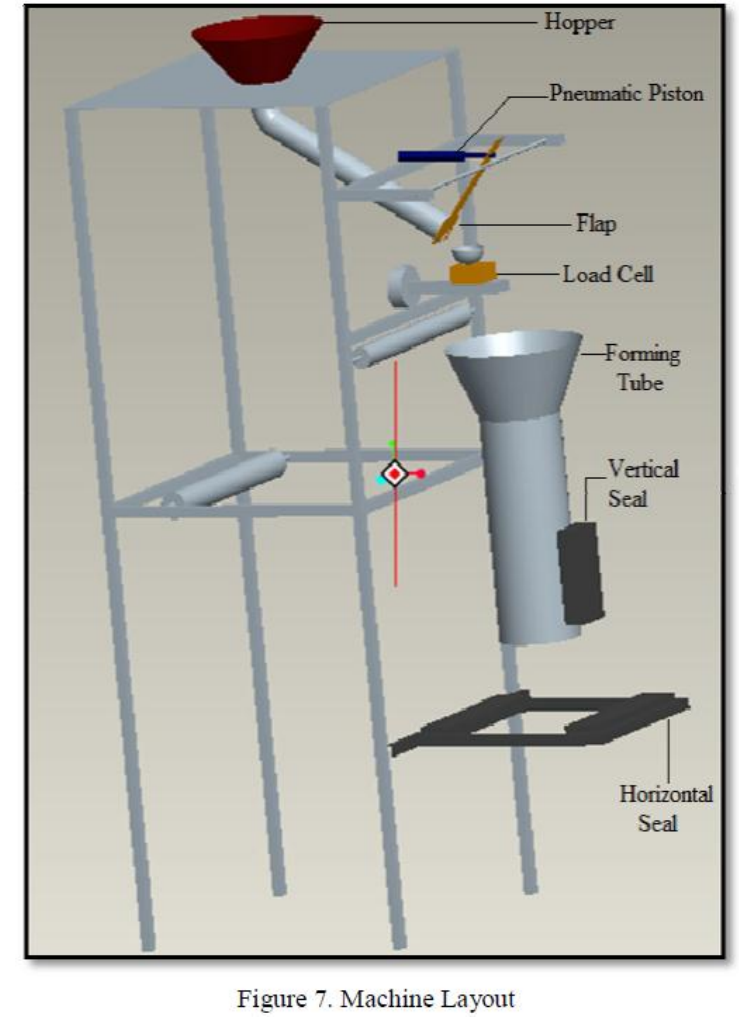

Heat sealers are mounted vertically and horizontally to make vertical and horizontal seals. Two horizontal sealers are placed to make the bottom and top seals of the pouch. These heat sealers are actuated by pneumatic cylinders. A set of draw rollers are mounted vertically on the forming tube which pull the formed plastic downwards. These rollers are rotated with the help of a motor. A cutter is placed between the horizontal seals to separate two consecutive bags from each other.

\subsection{Process Flow}

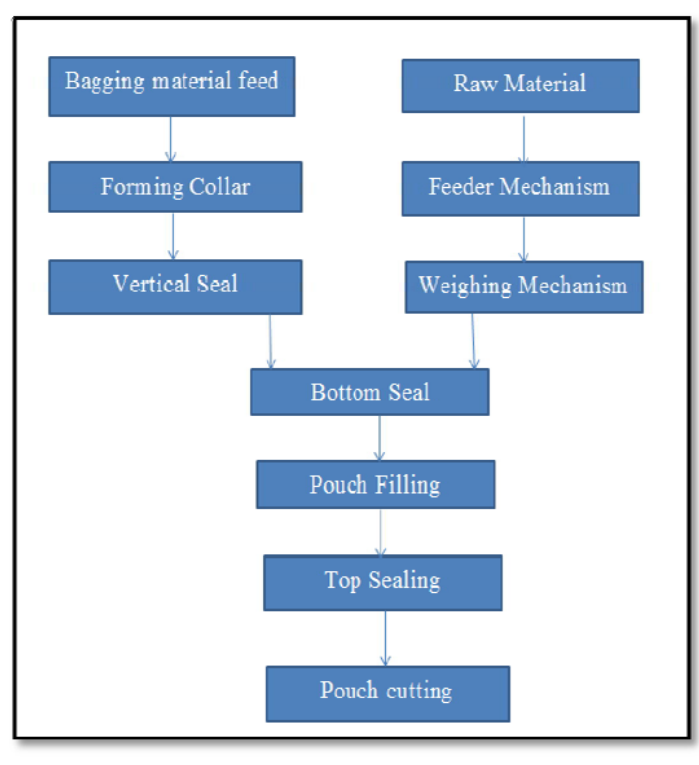

Figure 8. Process Flow 


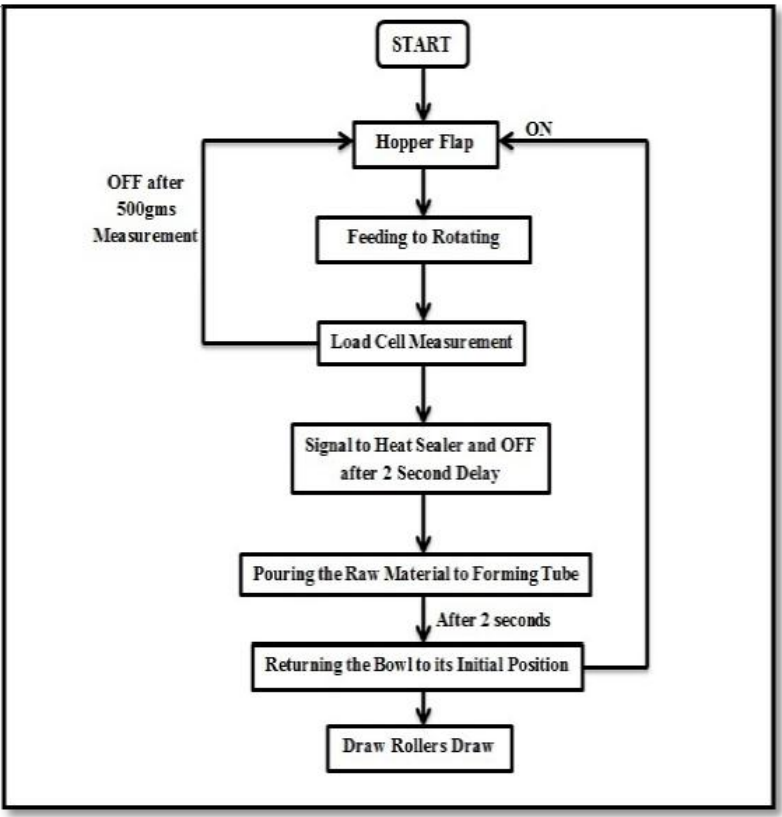

Figure 9. Algorithm

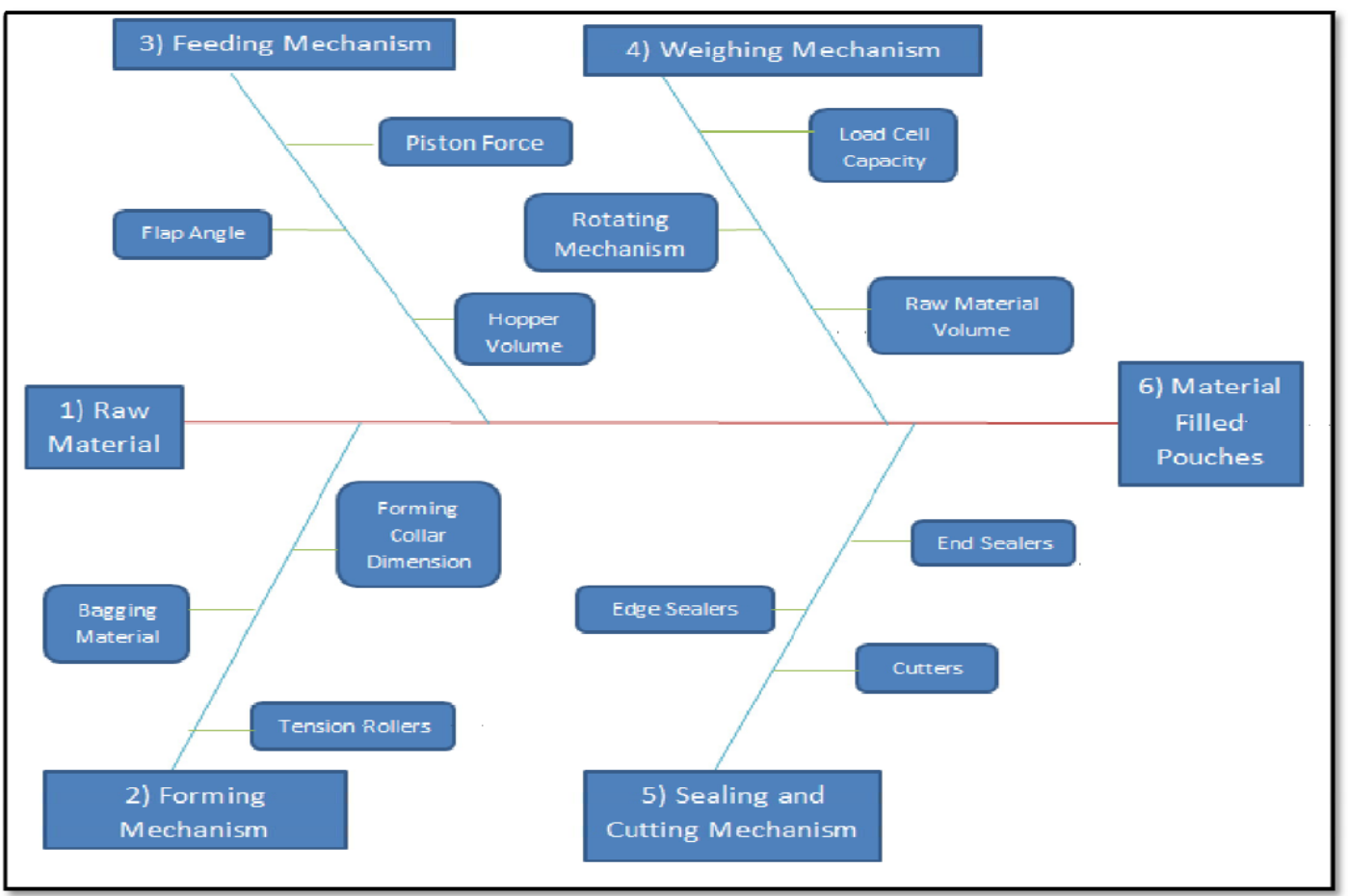

Figure 10. Cause effect Analysis (Fishbone Diagram)

\section{Cost Comparison}

Table 1 Cost Comparison between Various Systems

\begin{tabular}{|l|c|c|c|}
\hline & $\begin{array}{c}\text { Developed System } \\
(\tau)\end{array}$ & $\begin{array}{c}\text { Microcontroller based pouch } \\
\text { packing } \\
(\tau)\end{array}$ & $\begin{array}{c}\text { PLC based pouch packing } \\
(\tau)\end{array}$ \\
\hline Feeding Mechanism & 1,000 & 25,000 & 25,000 \\
\hline Forming Mechanism & 10,000 & 40,000 & 40,000 \\
\hline Weighing Mechanism & 4,000 & 10,000 & 10,000 \\
\hline $\begin{array}{l}\text { Sealing and cutting } \\
\text { Mechanism }\end{array}$ & 2,000 & 15,000 & 15,000 \\
\hline Automation & 3,000 & 30,000 & 30,000 \\
\hline Software & Free (C Programming) & Free (C programming) & $1,50,000$ (RS Logix, etc) \\
\hline Hardware & 5,000 & 30,000 (Programmer Kit) & $1,50,000$ \\
\hline Total & INR 25,000 & INR 1,50,000 (Approx.) & INR 4,50,000 (Approx.) \\
\hline
\end{tabular}


The system is divided in four main mechanisms Forming mechanism, Feeding mechanism, weighing mechanism and sealing mechanism automated by a microcontroller unit. Some of the mechanisms or operations are executed simultaneously. As shown in the diagram, the forming and feeding mechanism occur simultaneously as well as weighing and vertical sealing occur simultaneously. The top and bottom seals are done at the end.

\subsection{Algorithm}

The process algorithm explains the working of the system in detail. The material is initially fed in the hopper. The flap is opened by the pneumatic cylinder arrangement, the material falls in the rotating bowl which is measured by the load cell. The load cell sends a signal to the microcontroller after a fixed amount of material falls in to the bowl. The microcontroller immediately closes the flap. The signal to heat sealer piston is sent, the piston actuates and the heat sealer makes the vertical edge seal and the horizontal seal of the pouch, after a time delay the piston moves back to its original position which discontinues the contact of the heat sealer and the plastic pouch. After the seal the bowl rotates and the weighed material falls in the pouch which is open from top but sealed from bottom and vertically. The bowl returns back to its original position after a suitable time delay. When the bowl returns to its original position the flap of the hopper is opened and the cycle starts again. Meanwhile the draw rollers pull the formed plastic downwards by the length of one bag. The top seal of the pouch is done when the bottom seal of the next bag is made. Thus, a filled pouch separates from the next pouch when the top seal is made with the help of a cutter situated between the horizontal sealers.

\section{Future Scope}

The machine currently operates in four basic mechanisms- Feeding, Weighing, Forming and Sealing. The current design can pack one pouch at a time, a more advanced machine having multiple hoppers and feeders can be developed for packing multiple bags simultaneously.
A faster and more complex cup filler and feeder mechanism can be used so as to save time and obtain volumetric accuracy of the pouch to be filled. The system can be scaled against heavy loads.

\section{Conclusions}

Using currently available information and data on flexible automation techniques and mechatronics systems the automation of the machine has been developed. Due to a simple design and the use of low cost reliable components, the machine is developed in lesser cost as compared to other conventional machines. Hence, the Low cost automated Pouch Packing Machine is successfully developed which can benefit small Industries or enterprises. Safety of the workers is achieved as the machine requires very less human intervention.

\section{References}

Michal Kelemen, Tatiana Kelemenová, Ivan Virgala, d’ubicaMikováa, TomášLiptáka; Rapid Control Prototyping of Embedded Systems Based on Microcontrollers; (www.sciencedirect.com)

Emel Koçaka , Raif Bayırb; Project and group based learning and competition based evaluation in lesson of microcontroller applications (www.sciencedirect.com)

Shashank Lingappa M., Vijayavithal Bongale PLC Controlled Low Cost Automatic Packing Machine; International Journal of Advanced Mechanical Engineering ISSN 2250-3234 Volume 4.

Automation of VFFS Machine by Rockwell Automation; http://literature.rockwellautomation.com/idc/groups/ literature/documents/wp/oem-wp011_-en-p.pdf

Automation of Intermittent VFFS Machine using Allen Bradley Micro850 Programmable controllers http://literature.rockwellautomation.com/idcgroups/li terature/documents/wp/oem-wp011_-en-p.pdf

Introduction to PLC controllers(online): http://www.mikroe.com/old/books/plcbook/chapter1 /

Architecture and programming of 8051 MCU's (online): http://www.mikroe.com/chapters/view/64/chapter-1introduction-to-microcontrollers/ 